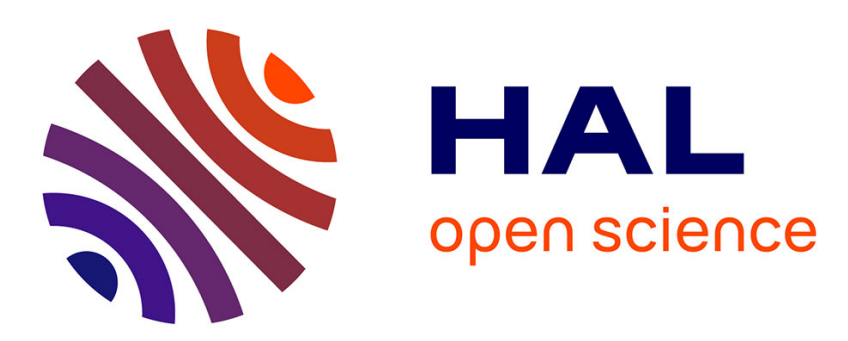

\title{
Une recherche sur la scolarité des enfants du voyage ou quelques conditions de transformations de pratiques institutionnelles par la recherche
}

Franck Bettendorff

\section{- To cite this version:}

Franck Bettendorff. Une recherche sur la scolarité des enfants du voyage ou quelques conditions de transformations de pratiques institutionnelles par la recherche. Revue Française de Pédagogie, 2017, pp.81 - 87. 10.4000/rfp.7029 . hal-03009494

HAL Id: hal-03009494

https://hal-univ-paris8.archives-ouvertes.fr/hal-03009494

Submitted on 17 Nov 2020

HAL is a multi-disciplinary open access archive for the deposit and dissemination of scientific research documents, whether they are published or not. The documents may come from teaching and research institutions in France or abroad, or from public or private research centers.
L'archive ouverte pluridisciplinaire HAL, est destinée au dépôt et à la diffusion de documents scientifiques de niveau recherche, publiés ou non, émanant des établissements d'enseignement et de recherche français ou étrangers, des laboratoires publics ou privés. 


\section{UNE RECHERCHE SUR LA SCOLARITÉ DES ENFANTS DU VOYAGE OU QUELQUES CONDITIONS DE TRANSFORMATIONS DE PRATIQUES INSTITUTIONNELLES PAR LA RECHERCHE}

\section{Franck Bettendorff}

\section{E.N.S. Editions | « Revue française de pédagogie »}

$2017 / 3 n^{\circ} 200$ | pages 81 à 87

ISSN 0556-7807

Article disponible en ligne à l'adresse :

https://www.cairn.info/revue-francaise-de-pedagogie-2017-3-page-81.htm

Distribution électronique Cairn.info pour E.N.S. Editions.

(C) E.N.S. Editions. Tous droits réservés pour tous pays.

La reproduction ou représentation de cet article, notamment par photocopie, n'est autorisée que dans les limites des conditions générales d'utilisation du site ou, le cas échéant, des conditions générales de la licence souscrite par votre établissement. Toute autre reproduction ou représentation, en tout ou partie, sous quelque forme et de quelque manière que ce soit, est interdite sauf accord préalable et écrit de l'éditeur, en dehors des cas prévus par la législation en vigueur en France. Il est précisé que son stockage dans une base de données est également interdit. 
ज्ञ FRANÇAISE

$\lessgtr \mathrm{DE}$

때 PÉDAGOGIE

\section{Revue française de pédagogie}

Recherches en éducation

$200 \mid 2017$

Recherche, politique et pratiques en éducation :

services rendus et questions posées d'un univers à

l'autre

\section{Une recherche sur la scolarité des enfants du voyage ou quelques conditions de transformations de pratiques institutionnelles par la recherche}

Research on the schooling of Traveller children or some conditions for the transformation of institutional practices through research

Franck Bettendorff

\section{(2) OpenEdition \\ Journals}

Édition électronique

URL : http://journals.openedition.org/rfp/7029

DOI : $10.4000 /$ rfp.7029

ISSN : 2105-2913

Éditeur

ENS Éditions

Édition imprimée

Date de publication : 30 septembre 2017

Pagination : 81-87

ISBN : 979-10-362-0124-0

ISSN : 0556-7807

Distribution électronique Cairn

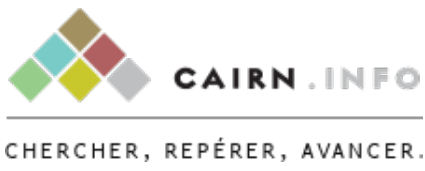

Référence électronique

Franck Bettendorff, «Une recherche sur la scolarité des enfants du voyage ou quelques conditions de transformations de pratiques institutionnelles par la recherche », Revue française de pédagogie [En ligne], 200 | 2017, mis en ligne le 01 janvier 2023, consulté le 10 avril 2019. URL : http://

journals.openedition.org/rfp/7029; DOI : 10.4000/rfp.7029 


\title{
Une recherche sur la scolarité des enfants du voyage ou quelques conditions de transformations de pratiques institutionnelles par la recherche
}

\author{
Franck Bettendorff
}

\begin{abstract}
Afin d'éclairer les services rendus par une recherche de sociologie de l'éducation sur des pratiques institutionnelles, cette contribution présente une recherche doctorale menée par un enseignant auprès des enfants du voyage, devenu chercheur. L'article croise des cadres théoriques issus de la sociologie des inégalités scolaires et des résultats d'une enquête de terrain dans un département qui a vu se développer pendant plus de dix ans une politique coordonnée de scolarisation des enfants du voyage. Cette recherche permet de mettre en évidence quelques conditions de transformation de pratiques institutionnelles par la mise en présence des catégories et résultats de la recherche avec la réflexion d'acteurs de terrain dans des situations de formations ou d'activités professionnelles.
\end{abstract}

Mots-clés (TESE) : sociologie de l'éducation, résultats de recherche, formation des enseignants, besoins éducatifs particuliers, groupe ethnique

Éclairer la question des «services rendus» par la sociologie de l'éducation aux pratiques des institutions et de leurs agents nécessite en préalable de rappeler que les champs heuristiques et praxiques se distinguent nettement par leurs finalités, objets et méthodes. La fameuse citation de Marx : «les philosophes n'ont fait qu'interpréter le monde : il s'agit de le transformer» (Marx \& Hengel, 1845) souligne la forte séparation de certains champs de savoirs d'avec la praxis. Certes, des types de recherches telles que la recherche-actionformation et les recherches en croisement des savoirs (Cornerotte, Ferrand \& Galvani, 2008, p. 22) intègrent des transformations mutuelles au cours du processus de recherche. Toutefois, pour des épistémologies plus radicalement fondées sur une rupture entre la connaissance et l'action, comme la sociologie critique, la 
transformation des pratiques par les objets de recherche n'a rien d'évident a priori. Ajoutons un second point de départ essentiel : I'hypothèse de spécificité des objets et des pratiques en jeu. II ne nous semble pas possible de conduire une réflexion générale sur les possibilités de transformation d'un univers par l'autre, en dehors de tout contexte scientifique et pratique. Notre hypothèse sera que c'est en référence à un objet, des questionnements et des résultats particuliers et un contexte institutionnel ou professionnel également particulier que peuvent être examinées les conditions de transformations réciproques.

L'argumentation qui suit est donc située et repose sur une recherche doctorale conduite à partir d'une insertion professionnelle longue dans un domaine tout à fait spécifique : la scolarisation des enfants du voyage'. L'entrée en thèse a été l'occasion de mettre au second plan certaines problématiques professionnelles en vue de la construction d'un objet de recherche. Cette transformation a nécessité un ensemble d'opérations qui, analysées, nous permettent aujourd'hui plus largement de contribuer à penser les conditions d'un apport de la sociologie de l'éducation aux pratiques institutionnelles et professionnelles.

Nous nous proposons donc d'examiner les conditions de rencontre entre un objet de recherche et des pratiques institutionnelles concernant les difficultés scolaires d'élèves appelés les enfants du voyage. Parler de rencontre implique une mise en dialogue, voire une mise en présence de termes distincts, sinon opposés. Dans notre cas précis de recherche par un doctorant qui est aussi un professionnel, de quelle rencontre s'agit-il ? Afin de répondre à cette interrogation, il s'agit d'abord de contextualiser notre réflexion en décrivant quelques dimensions de notre objet de recherche : questionnements, cadre théorique et résultats, puis dans un second temps de décrire quelques mises au travail qui ont eu cours au sein d'instances institutionnelles aux niveaux national puis départemental dans lesquelles nous avons été impliqué comme chercheur et enseignant. Nous allons à cet effet nous focaliser sur un aspect du questionnement de thèse, les catégories scolaires, et observer comment sa mobilisation dans les instances décrites plus loin a pu provoquer ou non des déplacements dans les discours et pratiques des agents. À partir de cette observation, nous tenterons

1 Tout en gardant par ailleurs un statut d'enseignant spécialisé pour ce même public scolaire, nous conduisons donc un travail de thèse depuis l'automne 2014. de dégager quelques conditions de transformation d'un champ par l'autre.

\section{L'enquête : mise en contextes, cadrage théorique et premiers résultats}

L'enquête de terrain sur laquelle s'appuie notre réflexion s'est déroulée sur deux années, de mars 2015 à juin 2017, dans un département où la scolarisation des enfants du voyage ou EFIV ${ }^{2}$ a fait l'objet d'une politique coordonnée depuis 2005 à l'initiative de I'Inspection académique. Il s'agit d'une enquête qualitative que l'on peut situer dans le cadre de la sociologie de l'éducation réalisée principalement par entretiens semi-directifs auprès d'élèves, de parents d'élèves et d'agents de dix établissements scolaires (principaux, vie scolaire, assistantes sociales scolaires, enseignants), mais aussi à travers une participation observante et l'analyse de journaux d'enseignants du dispositif, tous concernés à différents titres par la catégorie institutionnelle enfant du voyage. Enfin, l'enquête prend en compte un certain nombre d'écrits institutionnels (circulaires, rapports et comptes rendus nationaux ou départementaux).

Le premier ensemble de résultats concerne la catégorisation mobilisée. La circulaire ministérielle de 2012 affiche explicitement un objectif de «droit commun», c'est-à-dire « une scolarisation et une scolarité dans les mêmes conditions que les autres » (MEN, 2012, p. 1). Ce faisant, elle désigne implicitement un public scolaire spécifique, un public-problème (Dukic \& Dhume, 2011, p.56), auquel une attention particulière doit être portée, principalement quant à la fréquentation scolaire et à I'assiduité. Au niveau départemental et cela dès 2005, le dossier de la scolarisation des enfants du voyage a fait l'objet de la préoccupation d'un comité de pilotage dédié à ce public au même titre que d'autres élèves à besoins éducatifs particuliers pour lesquels la finalité annoncée est la scolarisation en établissement ordinaire ${ }^{3}$. Le Ministère tout comme l'Inspection académique mobilisent donc une distinction commune, entre les figures de l'ordinaire et du particulier. La scolarisation

2 Enfants issus de Familles Itinérantes et de Voyageurs (MEN, 2012). Par commodité et parce que enfants du voyage est encore en usage dans les établissements scolaires, nous utiliserons ici les deux dénominations enfants du voyage et EFIV, même si elles ne sont pas totalement équivalentes.

3 Inspection académique, compte rendu du groupe d'appui du vendredi 7 octobre 2005 
de droit commun ou l'inclusion est le principe politique et la scolarisation adaptée le moyen censé concilier provisoirement les deux termes ${ }^{4}$.

Comment saisir la portée de cette distinction? Quel cadre théorique de sociologie peut-on mobiliser? Pierre Bourdieu a en effet théorisé les notions d'effets de champs, c'est-à-dire comment les catégorisations visent à distinguer, voire hiérarchiser des groupes humains et ce faisant génèrent des effets de corps (Bourdieu, 1985). On peut également interroger la référence à des élèves particuliers en tant qu'elle constitue une opération de marquage social (Brekhus, 2005, p. 246). Ces élèves littéralement extraordinaires, par un effet de contraste, deviennent marqués dans les discours de la circulaire et du comité de pilotage local, en opposition aux élèves ordinaires qui demeurent non marqués, voire invisibilisés. Les catégories scolaires fondées sur des particularisations agissent potentiellement en essentialisant des publics cibles. En effet, dans le cas de la catégorie EFIV, la circulaire définit des élèves dont les familles ont un «mode de relation discontinu à l'école» (MEN, 2012, p. 1). Or, la circulaire traite tout autant de difficultés posées par un mode de vie itinérant que de particularités culturelles et d'appartenance à des communautés, comme l'indiquent par exemple les références aux craintes familiales vis-à-vis du collège ou encore à des traditions. Les élèves et leurs familles sont supposés avoir des rapports à l'école spécifiques, teintés de méfiance (Liégeois, 2007, p. 174). En s'appuyant sur la notion de rapport à l'école (Charlot, Bautier \& Rochex, 1992, p. 185), notre enquête montre que même si demeurent dans notre population quelques spécificités dans le rapport à l'école, on ne peut plus parler de méfiance vis-à-vis de l'école mais au contraire d'intérêt. Les élèves et les familles souhaitent que leurs enfants apprennent à lire et à écrire et se saisissent de l'école pour faciliter une insertion professionnelle.

On peut donc interroger la pertinence de la catégorie EFIV. Que signifie la désignation d'un public en référence à une origine ou à une appartenance communautaire? Elle peut être décrite avec la notion d'ethnicisation (Bonnéry, 2006, p. 2 ; Dukic \& Dhume, 2011, p. 55-56; Cossée, 2010, p. 162). Notre enquête de terrain tend à confirmer cette première analyse en terme d'ethnicisation d'un public-problème. Nous avons pu observer

4 MEN, 2012, p. 1 : «L'inclusion dans les classes ordinaires constitue la modalité principale de scolarisation. Elle est le but à atteindre, même lorsqu'elle nécessite temporairement des aménagements et des dispositifs particuliers». par exemple que des procédures de contrôle de l'assiduité ou des emplois du temps sont modifiées en référence à l'appartenance à ce public selon des modalités différenciées suivant les collèges. À propos d'une approche ethnicisante du contrôle des absences, citons par exemple une assistante sociale scolaire d'un collège public accueillant des enfants du voyage depuis 2009 :

ASS : mais je trouve que par moment ça vient plus de la vie scolaire.

Enquêteur : vous voulez dire : ils feraient pas remonter? ASS : non, ils font plus remonter. C'est plus facile de faire remonter euh; pour les élèves de la communauté des gens du voyage.

Si la notion d'ethnicisation est utile dans un premier temps pour décrire des distinctions, voire des régimes différents entre élèves, elle ne suffit pas pour autant pour analyser les mécanismes qui conduisent les agents à installer différents régimes de règles et de procédures en fonction d'une catégorisation comme celle d'enfants du voyage.

Le concept de classification du sociologue Basil Bernstein permet de penser les catégorisations déployées au sein des institutions scolaires comme des frontières, des séparations, entre des espaces actualisant avec des discours, pratiques et agent distincts, l'exercice du pouvoir (Bernstein, 2007, p. 27-29). L'instance qui détient l'autorité, ici le ministère de l'Éducation nationale, définit et crée les prérogatives, les champs de compétences et leurs modalités. Au sujet des enfants du voyage, la circulaire de 2012 rappelle l'autorité du Ministère : "Les orientations et les conditions générales de scolarisation des enfants de familles itinérantes et de voyageurs sont définies au niveau national ». Que dit par la suite ce texte des classifications dans ce qu'elles fondent des divisions sociales du travail? En rappelant le cadre de référence national, il institue au niveau académique un chargé du dossier "élèves issus de familles itinérantes et de voyageurs » et au niveau départemental un chargé de mission sous l'autorité directe de l'inspecteur d'académie. Ainsi, tout en rappelant la finalité du droit commun, la circulaire crée la possibilité d'un encadrement spécifique des scolarités des enfants du voyage. Le coordonnateur départemental en devient le pivot. Le cadrage mis en place pourra mobiliser des procédures particulières et des personnels dédiés qui ne dépendent pas des inspecteurs de circonscription ou des principaux de collège. On voit également apparaître un ensemble de procédures spécifiques : «Des dispositifs sont élaborés localement en vue de faciliter la scolarisation au collège (identification des élèves, 
dialogue suivi avec les parents, journées de visite du collège, pré-inscriptions, etc.) 》, mais aussi des personnels à vocation plus ou moins exclusive : «le médiateur scolaire et les personnes chargées de l'accueil des familles et du suivi de la scolarité des élèves dans les établissements et les écoles» ou des " professeurs relais» ou des «unités pédagogiques spécifiques».

\section{Les effets de la recherche au travers de deux situations professionnelles}

Les développements ci-dessus ont été l'occasion d'élaborer des questionnements et résultats d'une approche critique en sociologie de l'éducation sur un ensemble de pratiques institutionnelles pour des élèves concernés par la catégorie EFIV. II convient maintenant dans une seconde partie d'examiner les conditions et les possibilités des transformations possibles des pratiques par une recherche sur ces mêmes pratiques. Nous conduirons cette réflexion selon deux axes. Une première mise en débat de la catégorie elle-même dans une instance de formation, à l'aide des notions de marquage et d'ethnicisation. Ensuite, nous examinerons comment les catégories énoncées par les autorités fonctionnent comme des séparations symboliques et structurent l'ordre social. Selon Bernstein, à l'échelle institutionnelle, les séparations fonctionnent comme des moyens de gérer les contradictions et les dilemmes. À l'échelle des agents, elles fonctionnent comme des systèmes de défense (Bernstein, 2007, p. 30-31). Dans la réflexion sur la transformation des pratiques, on peut interroger le degré de questionnement possible des catégories. II est donc nécessaire de penser plus précisément les lieux et conditions d'élaboration de questionnements professionnels à partir de travaux de recherche potentiellement vecteurs de changement.

Pour cela, nous ferons référence à deux instances, dont une à l'échelle nationale et l'autre départementale, où comme chercheur, mais dans un exercice professionnel, nous avons pu faire l'expérience de déplacements de discours et pratiques, mais aussi de résistances.

La première instance appartient à I'Institut de La Salle qui dépend du réseau des Frères des Écoles Chrétiennes. Il s'agit actuellement d'un groupe chargé de concevoir les formations nationales de l'Enseignement catholique pour les enseignants auprès des enfants du voyage, des enfants migrants et d'autres enfants en difficulté scolaire. Initialement appelé groupe de pilotage d'action-recherche du réseau La Salle France pour les besoins éducatifs particuliers. Historiquement, la création de cette instance est étroitement liée à un enseignement original auprès de communautés Tsigane ou Gens $d u$ voyage. Les premières antennes scolaires mobiles de l'enseignement privé ont été créées par les Frères des Écoles Chrétiennes dans les années $1980^{5}$. Ces antennes ont été soutenues via la création de l'ASET dont le nom porte la marque de l'identité Tsigane ${ }^{6}$. Répondre à un besoin de scolarisation pour des enfants très peu scolarisés et développer un savoir professionnel spécifique sur les Tsiganes et Voyageurs ont été deux dimensions dès le départ étroitement associées. L'historique des noms des formations nationales d'enseignants organisées par le réseau lassalliens entre 1989 à 2010 montre que, sur quarante-cinq titres de sessions, quinze contiennent une référence explicite aux Tsiganes ou Gens du voyage. Quelques exemples : en 2010, «Bilinguisme, construction de la langue, difficultés de l'écrit chez les enfants du voyage»; en 2001, «Approche psychopédagogique dans la construction des apprentissages chez les enfants Tsiganes»; en 1995, "Culture tzigane»; en 1990, "Approche socio-culturelle des Tsiganes ». Revenons au groupe de pilotage qui existe depuis fin 2002. II se réunit pour concevoir, organiser et évaluer des sessions de formations annuelles. Il est composé de quatre enseignants élus parmi leurs pairs et placés sous la responsabilité de l'Institut de La Salle à Paris. Nous y prenons place en 2013 en tant qu'enseignant et en 2014, nous débutons notre doctorat de sciences de l'éducation. Or, après une première approche de la question dans un cadre anthropologique, nos travaux nous conduisent à intégrer une analyse sur l'école et les milieux populaires (Thin, 1998; Millet \& Thin, 2012; Perier, 2005). Informés de notre enquête, les autres membres du groupe de pilotage ont pu eux-mêmes partager leurs questionnements sur l'identité Tsiganes et voyageurs de ces formations. La question de la catégorie scolaire a donc été posée et travaillée. Pour illustrer la transformation opérée par le groupe, relevons ici une évolution des titres des formations depuis 2013, année où l'expression «du voyage » est utilisée pour la dernière fois. On peut constater un déplacement de dénominations mêlant caractéristiques ethniques et

5 Antenne Scolaire Mobile (ASM) désigne un dispositif d'enseignement comprenant un enseignant et un véhicule utilitaire aménagé en salle de classe.

6 Association d'Aide à la Scolarisation des Enfants Tsiganes et autres jeunes en difficulté. 
prise en compte du nomadisme ("enfants du voyage» ou «de passage ») à des élèves décrits par des caractéristiques en lien avec les apprentissages. En 2014 et 2015, la formation concerne «les enfants de passage», en 2016, "les enfants éloignés de l'école», en 2017, «pour des élèves dont le français est langue de scolarisation » et enfin en 2018 «élèves en rupture avec les apprentissages».

Cette brève description illustre la possibilité d'un déplacement catégoriel au sein d'une instance de formation. Peut-on en identifier quelques conditions? Un questionnement partagé et non imposé avec d'autres professionnels, la possibilité donnée par l'institution porteuse de laisser émerger de nouveaux questionnements et enfin la présence dans le groupe d'un chercheur, situé comme professionnel. Sur ce dernier point, le fait que les échanges puissent emprunter les mots mêmes des professionnels, au sein également de préoccupations de professionnels, est probablement une condition intéressante. Dans ce cadre, le rôle du chercheur-enseignant-formateur a consisté à formuler de façon élaborée scientifiquement des contradictions déjà pressenties de façon plus ou moins intuitive par d'autres enseignants, voire à contribuer à délégitimer des doxas institutionnelles.

La seconde instance est située à l'échelle du département d'enquête. Il s'agit d'une équipe d'enseignants et d'assistants pédagogiques spécialisés pour les enfants du voyage. Cette équipe appelée localement dispositif EFIV relève d'un partenariat local entre l'Éducation nationale et l'Enseignement catholique ${ }^{7}$. Un premier volet du travail du dispositif consiste à suivre des enfants ou des jeunes pas ou peu scolarisés, ou très absents de leurs classes, pour les inciter et les aider à rejoindre des établissements scolaires. Cela implique pour les enseignants de créer des relations suivies avec les familles en question. D'autre part, le dispositif répond à des demandes d'aide d'écoles et de collèges qui rencontrent des difficultés de tout ordre avec des enfants du voyage. Selon les cas, le dispositif peut être amené à prendre en charge une part d'enseignement pour ces élèves. II a aussi un rôle d'information des enseignants, agents de vie scolaire et directions d'établissements publics ou privés sur les modalités de prise en charge des enfants du voyage. Le dispositif se réunit régulièrement pour

7 II serait trop long ici de montrer en quoi ces enseignants prennent place dans un dispositif à la fois plus large et plus complexe. Nous garderons ici le terme dispositif qui désigne l'équipe d'enseignants et d'assistants pédagogiques. échanger sur les réussites et obstacles rencontrés et coordonner ses pratiques.

Afin d'examiner plus avant le potentiel rôle transformateur de notre recherche, il convient de préciser quelle est notre place dans ce dispositif. En plus de notre service d'enseignement, nous avons coordonné entre 2011 et 2017 la partie enseignement privé du dispositif, c'est-à-dire une équipe de quatre enseignants. En complément de la responsabilité de l'attribution des suivis d'élèves, nous avons animé des réunions à partir des situations problématiques rencontrées dans les écoles et collèges. Le thème central de la réflexion répondait à un besoin professionnel : la construction des discours et pratiques institutionnelles favorisant inclusion, assiduité et apprentissage.

Or, lors de son institutionnalisation, à l'automne 2005, le dispositif était censé répondre à un constat de «blocages» de la part des familles vis-à-vis de l'école, une difficulté à s'approprier les «droits et devoirs» en lien avec I'instruction scolaire. La culture des familles est un élément d'explication et un lieu de travail : «comment travailler sur la culture des familles du voyage pour faire avancer la scolarisation de leurs enfants? » (MEN, 2012, p. 3, «Missions du groupe d'appui »). Pourtant, notre enquête de terrain montre que l'explication culturaliste ne tient pas. Nous l'avons déjà souligné : dix ans après cette impulsion de l'Inspection académique, l'analyse de nos entretiens montre qu'il y a hétérogénéité des rapports à l'école des familles. Pour la majorité d'entre elles, l'école est même un lieu utile pour apprendre à lire et à écrire et pour préparer un avenir professionnel. Nos résultats sont plutôt bien reçus dans le dispositif où des enseignants sont mal à l'aise avec la dimension ethnique. Toutefois, c'est en relation avec la notion d'inclusion que la rencontre entre le questionnement des catégories et les pratiques nécessite davantage examen. En effet, alors que I'Inspection académique a renouvelé régulièrement depuis 2005 l'injonction de l'inclusion en classe ordinaire, un certain nombre d'élèves restent exclusivement confiés au dispositif par des collèges. Malgré les sollicitations, des professeurs résistent à l'accueil de ces élèves dans leurs classes. Les motifs invoqués sont soit leur trop faible niveau scolaire (ils ne peuvent pas suivre l'enseignement dispensé), soit qu'ils ne sont pas assez assidus, soit qu'ils relèvent du dispositif enfants du voyage. Dans ce contexte, quelles transformations notre recherche produit-elle? Elle introduit dans les échanges entre les enseignants du dispositif et avec les enseignants des classes ordinaires la mise en question du marquage ethnique des élèves 
comme unique explication. Le seul marquage enfant du voyage d'un élève justifie-t-il en outre son affectation dans le dispositif? Ainsi, la mise au second plan de la dimension ethnique de la catégorie semble faire émerger la notion d'épreuve professionnelle. Scolariser un élève pour lequel le besoin d'attention, de préparation voire de modification des habitudes pédagogiques est élevé peut se révéler être une épreuve professionnelle trop importante. Dans certains cas, des professionnels semblent résister aux résultats de la recherche, en continuant de faire fonctionner le marquage des élèves ou l'ethnicisation, mais on peut se demander si ce sont des résistances en tant que persistances de logiques de catégorisation ou de répartition des rôles (prégnance des classifications)? La désignation de communautés marquées ne masque-t-elle pas des tentatives de reporter la responsabilité de l'épreuve sur des causes extérieures à l'école en invisibilisant des mécanismes internes?

Ce second exemple nous permet de formuler deux nouvelles conditions d'une transformation des pratiques par la recherche. Tout d'abord, dans le cas d'une enquête sur une population comme celle des enfants du voyage, qui constitue en milieu scolaire un groupe marqué, la centration sur les individus ou les situations les plus typiques peut contribuer à renforcer le marquage de la population (Brekhus, 2005, p. 252) et invisibiliser potentiellement des problèmes internes à l'institution. Par exemple, ce qu'on appelle dans l'Éducation nationale absentéisme n'est pas réservé aux enfants du voyage. Des difficultés à s'approprier certains apprentissages sont aussi rencontrées par les élèves de milieux populaires. La gestion des écarts de niveau importants dans une classe est aussi un problème assez commun. Si l'on n'y prête pas attention, une recherche sur un public particulier, en alimentant une catégorisation, peut donc contribuer à légitimer les classifications institutionnelles et renforcer des divisions entre l'ordinaire et le spécifique ${ }^{8}$. Une première condition de transformation des pratiques résiderait donc dans l'attention de la recherche aux problèmes ordinaires (et non pas particuliers) que mettent parfois en exergue des populations extraordinaires.

Une seconde condition serait dans le choix des objets de recherche en ce qu'ils remettent potentiellement en cause ou non les catégories et classifications institutionnelles. Un des objets de la sociologie est justement de questionner les significations attribuées à tel ou tel phénomène. Or, les agents ne peuvent pas aisément modifier les classifications qui sont à l'origine de leurs statuts et rôles car «les identités construites sont prises pour réelles, authentiques, constituantes, comme la source de l'intégrité» (Bernstein, 2007, p. 30). En fonction de ce qui vient d'être dit, il nous semble possible de formuler en hypothèse une dernière condition de transformation des pratiques : I'implication des différents niveaux de responsabilité dans la mise en œuvre de questionnements. Cette implication nécessiterait sans doute des espaces de concertation associant les différentes échelles et des chercheurs. Par exemple, lorsqu'en février 2017, le directeur académique adjoint responsable du dossier enfants du voyage affirme en réunion de dispositif que tous les enseignants doivent se charger des apprentissages des enfants du voyage, les membres du dispositif réagissent en marquant leur scepticisme et relèvent le besoin d'une intervention systématique de sa part auprès des chefs d'établissements ${ }^{9}$. Dans un contexte de faible nombre d'enfants du voyage répertoriés sur le département ${ }^{10}$ et de multiplicité des problèmes généraux comme l'absentéisme, la violence scolaire, etc., on peut faire l'hypothèse que traiter les questions posées par les enfants du voyage, en concertation avec des chercheurs, nécessite un réaménagement catégoriel et institutionnel plus général.

\section{Conclusion}

En conclusion, l'exposé qui précède vise à dégager quelques conditions d'une transformation de pratiques institutionnelles par des recherches en sociologie de l'éducation sur un sujet particulier : les difficultés scolaires des enfants du voyage. Nous avons considéré la catégorie institutionnelle EFIV comme un cas particulier de la métacatégorie élèves à besoins éducatifs particuliers. Cette catégorisation introduit des classifications, c'està-dire des séparations dans l'ordre institutionnel. Penser cette population scolaire comme objet d'un marquage social, d'une ethnicisation et de répartition du travail enseignant entre des élèves ordinaires et les enfants du voyage nous a permis d'examiner deux instances où nous avons été impliqué à la fois comme chercheur et comme enseignant. Les résultats de la recherche ont permis d'y faire évoluer la perception

9 Réunion d'harmonisation du dispositif EFIV du 6 février 2017.

10370 élèves sur l'ensemble du département répertoriés pour l'année 2014-2015. Données du coordonnateur départemental, mars 2015. 
ethnique du public scolaire. Sans nier les résistances rencontrées, celles-ci nous ont permis de formuler des conditions relevant de la recherche et des institutions en ce qu'elles favorisent l'émergence de nouveaux questionnements. Enfin, à partir de la distinction entre élèves ordinaires et élèves à besoins particuliers, nous avons pu examiner en quoi certains de nos résultats,

\section{Bibliographie}

BERNSTEIN B. (2007). Pédagogie, contrôle symbolique et identité : théorie, recherche, critique. Laval : Presses de I'Université de Laval.

BONNÉRY S. (2006). «La question de "l'ethnicité" dans l'École : essai de reconstruction du problème». Sociétés et jeunesses en difficulté, $\mathrm{n}^{\circ} 1$. En ligne : <http://sejed.revues.org/109> (consulté le 6 août 2016).

BOURDIEU P. (1985). «Effet de champ et effet de corps». Actes de la recherche en sciences sociales, vol.59, $\mathrm{n}^{\circ} 1$, p.73.

BREKHUS W. (2005). "Une sociologie de I'"invisibilité" : réorienter notre regard ». Réseaux, $\mathrm{n}^{\circ} 129-130, \mathrm{p} .243-272$.

CHARLOT B., BAUTIER É. \& ROCHEX J.-Y. (1992). École et savoir dans les banlieues et ailleurs. Paris : Armand Colin.

COSSÉE C. (2010). "L'impossible neutralité des sciences sociales face aux catégorisations militantes. "Tsiganes", "Gens du voyage", "Rroms" ou autres ethnonymes?». Migrations Société, n¹28, p.159-176.

DUKIC S. \& DHUME F. (2011). «À l'école avec les élèves roms, tsiganes et voyageurs». Cahiers pédagogiques, vol.21, p. 55-56. en questionnant la structure même de l'institution scolaire, rendent a priori difficile leur réception.

Franck Bettendorff Université Paris 8-Vincennes-Saint-Denis, CIRCEFT-ESCOL franck.bettendorff@wanadoo.fr

CORNEROTTE D., FERRAND D. \& GALVANI P. (2008). « Méthodologie ». In Groupes de recherche Quart monde-Université \& Quart monde Partenaire, Le croisement des savoirs et des pratiques. Paris : Éd. de l'Atelier, p. 21-23.

LIÉGEOIS J.-P. (2007). Roms en Europe. Strasbourg : Éd. du Conseil de l'Europe.

MARX K. \& ENGELS F. (1845). L'idéologie allemande. Première partie : Feuerbach. Traduction française de Renée Cartel et Gilbert Badia (1952). Paris : Les Éd. sociales.

MILLET M. \& THIN D. (2012). Ruptures scolaires : l'école à l'épreuve de la question sociale. Paris: PUF.

MINISTÈRE DE L'ÉDUCATION NATIONALE [MEN] (2012). «SCOlarisation et scolarité des enfants issus de familles itinérantes et de voyageurs ». Circulaire $n^{\circ} 2012-142$ du 2 octobre 2012. Bulletin officiel de l'Éducation nationale, 11 octobre, $\mathrm{n}^{\circ} 37 \mathrm{H}$.

PÉRIER P. (2005). École et familles populaires : sociologie d'un différend. Rennes : Presses universitaires de Rennes.

THIN D. (1998). Quartiers populaires : l'école et les familles. Lyon : Presses universitaires de Lyon. 